\title{
Development and Characterization of Monoclonal Antibodies against Nucleoprotein for Diagnosis of Influenza A Virus
}

\author{
Nguyen Hong Phuong ${ }^{1,2}$, Chaewon Kwak ${ }^{1,2}$, Chang-Kyu Heo ${ }^{3}$, Eun Wie Cho ${ }^{3}$, Jihyun Yang ${ }^{1 *}$, and Haryoung Poo ${ }^{1,2 *}$ \\ ${ }^{1}$ Infectious Disease Research Center, Korea Research Institute of Bioscience and Biotechnology, Daejeon 34141, Republic of Korea \\ ${ }^{2}$ Department of Biosystems and Bioengineering, KRIBB School of Biotechnology, University of Science and Technology, Daejeon 34113, \\ Republic of Korea \\ ${ }^{3}$ Rare Disease Research Center, Korea Research Institute of Bioscience and Biotechnology, Daejeon 34141, Republic of Korea
}

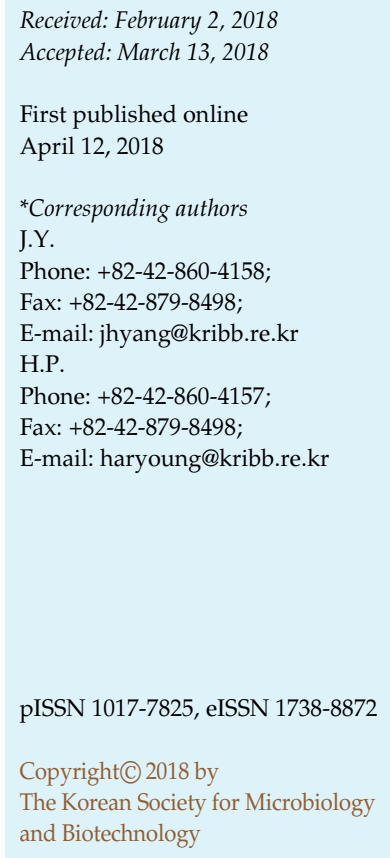

Influenza, which is a highly contagious disease caused by the influenza A virus, continues to be a major health concern worldwide. Although the accurate and early diagnosis of influenza virus infection is important for controlling the spread of this disease and rapidly initiating antiviral therapy, the current influenza diagnostic kits are limited by their low sensitivity. In this study, we developed several new influenza nucleoprotein (NP)-specific monoclonal antibodies (mAbs) and compared their sensitivity and specificity of those with commercially available anti-NP mAbs. Three mAbs, designated M24.11, M34.3, and M34.33, exhibited higher reactivities to recombinant NPs and A/Puerto Rico/8/1934 (H1N1) viral lysates compared with the commercial mAbs, as assessed using enzyme-linked immunosorbent assays. M34.3 and M34.33 showed higher reactivities with A/California/04/09 (pandemic H1N1) and A/ Philippines/2/82 (H3N2) viral lysates than the commercial mAbs. In contrast, M24.11 had marked reactivity with $\mathrm{H} 3 \mathrm{~N} 2$ but not with pandemic H1N1. Immunofluorescent confocal microscopy showed that the three mAbs effectively detected the presence of influenza virus in lung tissues of mice infected with A/Puerto Rico/8/1934. These results indicate that the newly developed M34.3 and M34.33 mAbs could be useful for the development of influenza diagnostics.

Keywords: Influenza A virus, nucleoprotein, monoclonal antibody, diagnosis, sensitivity

\section{Introduction}

The influenza A virus, which is a causative pathogen of highly infectious disease, is a negative-sense single-stranded RNA virus of the family Orthomyxoviridae. Since antigenic shift increases the emergence of novel influenza viruses, the 2009 pandemic influenza A virus subtype H1N1 resulted in globally severe morbidity and mortality [1-3]. Moreover, seasonal influenza A virus routinely causes half a million deaths worldwide and results in huge losses to global economies [4]. If we hope to control influenza virus infection, we crucially need a means for its accurate and early diagnosis [5]. This will enable the application of appropriate antiviral drugs and ameliorate the spread and severity of the disease.
The current diagnostics for influenza are broadly classified as protein- or nucleic acid-based assays [5, 6]. Protein-based diagnostics commonly use monoclonal antibodies (mAbs) to detect influenza virus antigens via various assays, including enzyme-linked immunosorbent assays (ELISAs), rapid influenza diagnostic tests (RIDTs), and direct fluorescent antibody tests. Nucleic acid-based diagnostics detect influenza virus-specific RNA sequences by various methods, such as reverse transcription-polymerase chain reaction, loop-mediated isothermal amplification-based assays, and sequencing-based tests. At present, the proteinbased assays, particularly RIDTs, are considered to be crucial point-of-care tests because they are easy to use and provide rapid results $[7,8]$.

The most common antigen target of commercially 
available influenza diagnostics is nucleoprotein (NP). The influenza surface antigens, like hemagglutinin (HA) and neuraminidase (NA), have such a high frequency of antigenic variation that their HA- and NA-specific mAbs have proven unsuitable for the development of influenza diagnostics [9]. NP, in contrast, is an internal antigen that is well conserved among the influenza A virus subtypes [5, 7, 10]. However, although anti-NP mAbs have been intensively developed for such applications, low clinical sensitivity remains the limitation of current influenza diagnostic kits $[5,11-13]$. Thus, we urgently need to develop more sensitive $\mathrm{mAbs}$ for the accurate and rapid diagnosis of influenza.

In the present study, we produced three mAbs against $\mathrm{NP}$ and investigated their sensitivity and specificity using various influenza A viruses. Compared with commercially available anti-NP mAbs, two of the generated mAbs, M34.3 and M34.33, showed more robust reactivity to several influenza A viruses, such as A/Puerto Rico/8/1934 (H1N1), A/California/04/09 (pandemic H1N1; pH1N1), and H3N2 A/Philippines/2/82. The third mAb, M24.11, had high reactivity to H1N1 and H3N2 but not pH1N1. These findings suggest that the newly developed M34.3 and M34.33 mAbs may be useful for the accurate and rapid diagnosis of influenza.

\section{Materials and Methods}

\section{Preparation of Recombinant NP (rNP) Antigen}

The full-length NP gene from influenza virus A/Puerto Rico/ 8/1934 (GenBank Accession No. CY105915) was chemically synthesized and inserted into the bacterial expression vector pET21a $(+)$ (Novagen, USA) to enable the expression of NP fused to a six-histidine tag. The recombinant plasmids were heat-shock transformed into Escherichia coli $\mathrm{C} 43$ and transformants were selected on a Luria-Bertani (LB) agar plate containing $100 \mu \mathrm{g} / \mathrm{ml}$ ampicillin (LBamp). A single colony was inoculated into $10 \mathrm{ml}$ of LBamp medium and incubated aerobically at $37^{\circ} \mathrm{C}$ for $16 \mathrm{~h}$. The cells were further cultured in a large volume of fresh LBamp medium at $37^{\circ} \mathrm{C}$ in a shaking incubator. When the cells reached an optical density at $600 \mathrm{~nm}\left(\mathrm{OD}_{600}\right)$ of approximately 0.4, rNP expression was induced by the addition of isopropyl $\beta$-D-1thiogalactopyranoside $(0.5 \mathrm{mM})$ and further incubation for $20 \mathrm{~h}$ at $20^{\circ} \mathrm{C}$. Cell pellets were harvested by centrifugation and rNP was purified as previously reported [14]. Briefly, the soluble fraction was obtained and histidine-tagged rNP was purified by affinity chromatography using nickel-nitrilotriacetic acid agarose (Qiagen, Germany) and size-exclusion chromatography using a HiLoad Superdex 200 column (GE Healthcare, UK). The purified NP antigen was confirmed by SDS-PAGE and quantified using a bicinchoninic acid protein assay kit (Pierce, USA). The rNP was approximately $57 \mathrm{kDa}$ in size.

\section{Viruses}

Influenza viruses A/Puerto Rico/8/34 (H1N1), A/California/ 04/09 (pH1N1), and A/Philippines/2/82 (H3N2) (provided by International Vaccine Institute, Korea) were grown in the allantoic cavities of 10-day-old embryonated chicken eggs for $48 \mathrm{~h}$ at $37^{\circ} \mathrm{C}$, as described previously [15]. Viruses were harvested in allantoic fluid by filtration through a $0.45-\mu \mathrm{m}$ filter and samples were stored at $-70^{\circ} \mathrm{C}$ until use. All viral experiments were performed under biosafety level $2+$ conditions.

\section{Mouse Immunization and Hybridoma Preparation}

Animal experiments were approved by the Institutional Animal Care and Use Committee of the Korea Research Institute of Bioscience and Biotechnology (KRIBB) (Approval No. KRIBBAEC-15131) and were performed according to the Guidelines for Animal Experiments of the KRIBB. Six-week-old female BALB/c mice purchased from Orient Bio (Korea) were housed in a specific pathogen-free facility at the KRIBB and allowed to acclimate for least 1 week before use. Mice were immunized with $10 \mu \mathrm{g}$ of purified rNP mixed with TiterMax Gold adjuvant (Sigma-Aldrich, USA) by footpad injection on days 0,7 , and 14 . Seven days after the last immunization, cells were isolated from popliteal lymph nodes and used to construct a B-cell hybridoma cell pool that produces anti-NP antibodies. Cell fusion and B-cell hybridoma selection were performed as previously described [16]. The reactivities of the antibodies produced by these hybridomas were determined by ELISA (see below). The isotype of each mAb was determined with an immunoglobulin isotyping kit (Roche Diagnostics, Germany). The mAbs were purified using protein $G$ columns (GE Healthcare, USA).

\section{ELISA}

MaxiSorp 96-well plates (Thermo Scientific, Denmark) were coated with rNP antigen ( 2 to $5 \mu \mathrm{g} / \mathrm{ml}$ ) in phosphate buffered saline (PBS) at $4^{\circ} \mathrm{C}$ overnight. The plates were incubated with $5 \%$ skim milk in PBS for blocking nonspecific antibody binding, washed, and then incubated with culture supernatants or the purified $\mathrm{mAbs}$ for $2 \mathrm{~h}$ at $37^{\circ} \mathrm{C}$ followed by horse-radish peroxidase (HRP)-conjugated anti-mouse IgG (Cell Signaling Technologies, USA) for $1 \mathrm{~h}$ at $37^{\circ} \mathrm{C}$. The plates were washed and developed with the chromogenic tetramethylbenzidine substrate (BD Biosciences, USA). The colorimetric reaction was terminated with $2 \mathrm{~N} \mathrm{H}_{2} \mathrm{SO}_{4}$, and the absorbance was measured at $450 \mathrm{~nm}$ with a Versamax microplate reader (Molecular Devices, USA). The commercially available anti-NP mAbs, C47G (Thermo Scientific, USA) and 9G8 (Santa Cruz Biotechnology, USA), were compared with the newly generated $\mathrm{mAbs}$. Viral lysates were prepared using an extraction buffer containing $50 \mathrm{mM}$ Tris (pH 7.5), $280 \mathrm{mM} \mathrm{NaCl}, 1 \%$ Triton X-100, $0.2 \mathrm{mM}$ EDTA, 10\% glycerol, $1 \mathrm{mM}$ dithiothreitol, and $1 \mathrm{mM}$ PMSF, as described previously [17-19].

\section{Western Blot Analysis}

Protein samples were separated by $12 \%$ SDS-PAGE (Bio-Rad, 


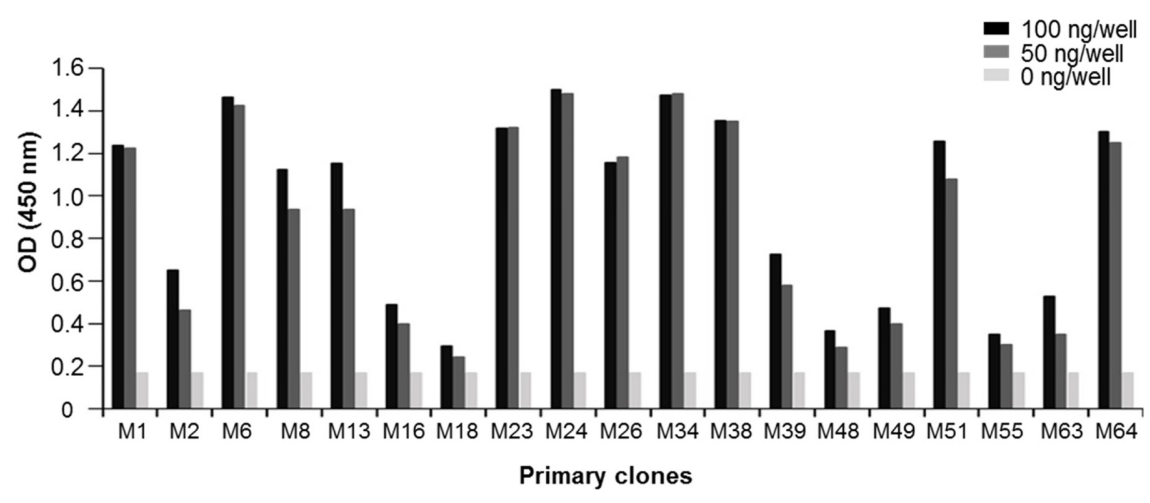

Fig. 1. ELISA-based comparison of the reactivity of Abs produced from primary clones.

ELISA plates were coated with $2.5 \mu \mathrm{g} / \mathrm{ml}$ recombinant nucleoprotein (rNP) for $12 \mathrm{~h}$ at $4^{\circ} \mathrm{C}$, blocked with $5 \%$ skim milk in PBST, washed, and incubated with Abs at 100 and $50 \mathrm{ng} /$ well followed by HRP-conjugated anti-mouse IgG. Reactivity to rNP was determined by ELISA and corrected OD values are shown.

USA) and transferred onto a polyvinylidene fluoride (PVDF) membrane (Bio-Rad, USA). After being blocked with 5\% skim milk in PBS containing 0.05\% Tween-20, the blots were probed with anti-NP mAbs and then incubated with HRP-conjugated goat anti-mouse IgG. The blots were washed and the immunoreactive bands were developed with an ECL western blotting detection solution (iNtRON Biotechnology, Korea) and visualized using an Azure C300 western blot imager (Azure Biosystems, USA).

\section{Fluorescent Immunohistochemistry}

Six-week-old female C57BL/ 6 mice were challenged intranasally with 10 times the $50 \%$ lethal dose $\left(\mathrm{LD}_{50}\right)$ of PR8 virus. Four days after this viral challenge, lung tissues were excised from the middle lobes. The samples were fixed, paraffin embedded, and sectioned. Tissue slides were stained with anti-NP mAbs and then incubated with Alexa Fluor 488-conjugated goat anti-mouse IgG (Invitrogen, USA). Nuclei were counterstained with 4',6-diamidino2-phenylindole (DAPI, $10 \mu \mathrm{g} / \mathrm{ml}$ ) for $3 \mathrm{~min}$. The stained sections were washed with PBS and observed using an Olympus FluoView laser scanning confocal microscope (Carl Zeiss, Germany).

\section{Results}

\section{Production and Characterization of Anti-NP mAbs}

To produce NP-specific mAbs, we immunized the footpads of BALB/c mice with rNP mixed with TiterMax Gold adjuvant on days 0,7 , and 14 . Seven days after the

Table 1. Isotyping of anti-NP mAbs.

\begin{tabular}{ccc}
\hline mAbs & Isotype & Light chain \\
\hline M24.11 & IgG1 & Kappa \\
M34.3 & IgG1 & Kappa \\
M34.33 & IgG1 & Kappa \\
\hline
\end{tabular}

final administration, hybridoma clones were prepared by fusing myeloma cells with popliteal lymph node cells obtained from the immunized mice. Reactivity to rNP was assessed by ELISA, which showed that two primary clones, M24 and M34, showed higher OD values against rNP compared with the other primary clones (Fig. 1). Thus, we selected the hybridomas for single-clone screening and identified three monoclones (M24.11, M34.3, and M34.33) as having robust reactivity to $\mathrm{rNP}$. These mAbs were used for further experiments. Their isotypes were determined using commercially available kits, which revealed that all three selected mAbs were IgG1 kappa-chain isotypes (Table 1). Next, we investigated whether each mAb

A

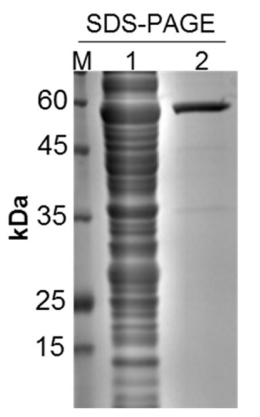

B
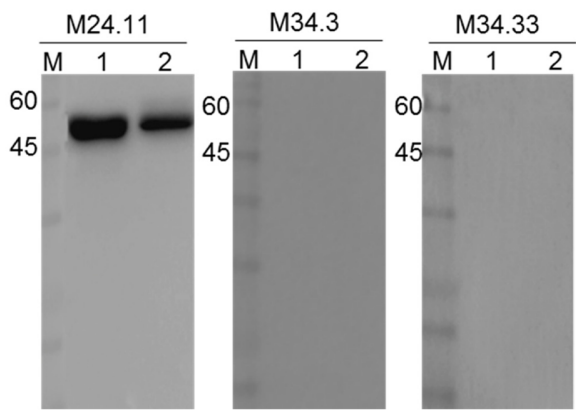

Fig. 2. Western blot analysis using the generated mAbs to recognize NP.

NP-containing E. coli lysates (lane 1) and recombinant NPs (lane 2) were separated by (A) 12\% SDS-PAGE under reducing conditions and (B) transferred to PVDF membranes. The membranes were probed with three mAbs, M24.11, M34.3 and M34.33 (1 $\mu \mathrm{g} / \mathrm{lane})$, followed by HPR-conjugated anti-mouse IgG. Lane M indicates protein markers $(\mathrm{kDa})$. 
A

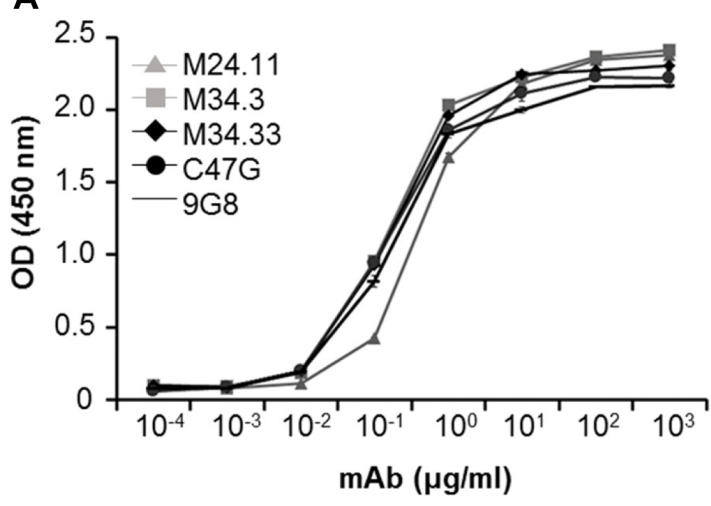

B

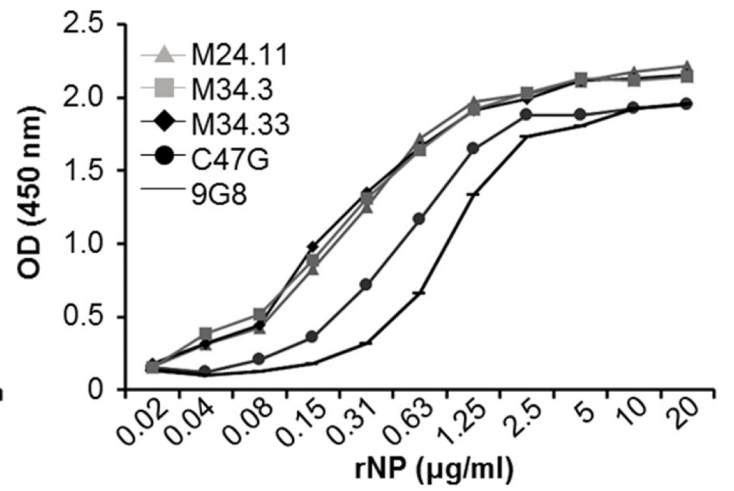

Fig. 3. ELISA-based comparison of the reactivities of the anti-NP mAbs.

(A) ELISA plates were coated with $2.5 \mu \mathrm{g} / \mathrm{ml}$ recombinant nucleoprotein (rNP), blocked with $5 \%$ skim milk in PBST, and washed with PBST. Newly generated $\mathrm{mAbs}$ were added in 10 -fold serial dilutions. The $\mathrm{EC}_{80}$ was determined using Origin 8.5 software. (B) rNP was coated in a series of 2-fold dilutions and the mAbs were applied at $100 \mathrm{ng} /$ well. Two commercial NP-specific mAbs, C47G and 9G8, were used for comparison. Corrected OD values are shown and are expressed as the mean \pm standard error.

recognizes a linear or conformational epitope of the NP antigen. Western blotting revealed that M24.11 had a highintensity reaction to rNP antigen, whereas M34.3 and M34.33 did not (Fig. 2). These results indicate that $\mathrm{mAb}$ M24.11 may bind to a linear epitope, whereas M34.3 and M34.33 may react to conformational epitopes of NP.

\section{Binding Affinities of the mAbs against rNP Antigen}

To measure the apparent binding affinities of the generated mAbs, we performed ELISA using 10-fold serial dilutions of the $\mathrm{mAbs}$ on rNP-coated plates. The commercially available anti-NP mAbs C47G and 9G8 were used for comparison. Based on the binding curves shown in Fig. 3A, we determined the binding affinities by calculating the $80 \%$ maximal effective concentration $\left(\mathrm{EC}_{80}\right)$ for each $\mathrm{mAb}$. The $\mathrm{EC}_{80}$ values of M34.3 and M34.33 (50 pM and $62 \mathrm{pM}$ ) were almost 2-fold lower than that of M24.11 $(129 \mathrm{pM})$. Under the same conditions, the $\mathrm{EC}_{80}$ values of commercial C47G and 9G8 were $100 \mathrm{pM}$ and $160 \mathrm{pM}$, respectively (Table 2). Thus, the $\mathrm{EC}_{80}$ values of our mAbs were subnanomolar, placing their binding within the range

Table 2. $\mathrm{EC}_{80}$ of anti-NP mAbs.

\begin{tabular}{cc}
\hline $\mathrm{mAbs}$ & $\mathrm{EC}_{80}(\mathrm{pM})$ \\
\hline $\mathrm{M} 24.11$ & 129 \\
$\mathrm{M} 34.3$ & 50 \\
$\mathrm{M} 34.33$ & 62 \\
$\mathrm{C} 47 \mathrm{G}$ & 100 \\
9G8 & 160 \\
\hline
\end{tabular}

of most approved antibodies. To further evaluate the binding affinities of the NP-specific mAbs, we performed ELISA using a consistent amount of $\mathrm{mAb}$ and plates coated with 2-fold serial dilutions of rNP. The obtained binding curves showed that the binding affinities of M24.11, M34.3, and M34.33 were higher than those of C47G and 9G8 (Fig. 3B). Our results therefore suggest that M34.3 and M34.33 could potentially be used in developing ELISAbased diagnostic kits.

\section{Specificity of NP-Specific mAbs against Influenza A Viruses}

Because most influenza A viruses circulating among humans are subtypes H1N1 and H3N2 [2], we used ELISA to investigate the specificity of the generated anti-NP mAbs to viral lysate antigens from A/Puerto Rico/8/34 (H1N1), A/California/04/09 (pH1N1), and A/Philippines/2/82 (H3N2). Compared with the commercial mAb C47G, the generated mAbs (M24.11, M34.3, and M34.33) bound somewhat more strongly to $\mathrm{H} 1 \mathrm{~N} 1$ viral lysate antigens (Fig. 4A). When bound to pH1N1 viral lysate antigens, M34.33 and M34.3 exhibited higher OD values on ELISA compared with $\mathrm{C} 47 \mathrm{G}$, whereas M24.11 showed the lowest OD value among them (Fig. 4B). With respect to crossreactivity, ELISA revealed that M24.11 (0.38 to $3 \mu \mathrm{g} / \mathrm{ml})$ showed the highest OD value against the H3N2 viral lysate antigens, followed by M34.3 and M34.33. C47G showed the lowest OD value under the same conditions (Fig. 4C). Our results indicate that the NP-specific mAbs M34.3 and M34.33 could potentially be useful for diagnosing influenza A virus infection. 
A

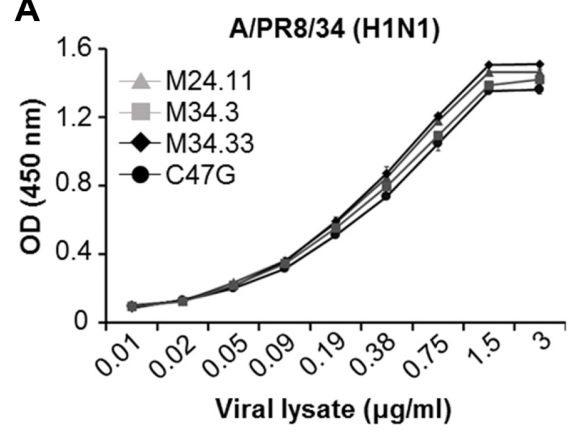

B

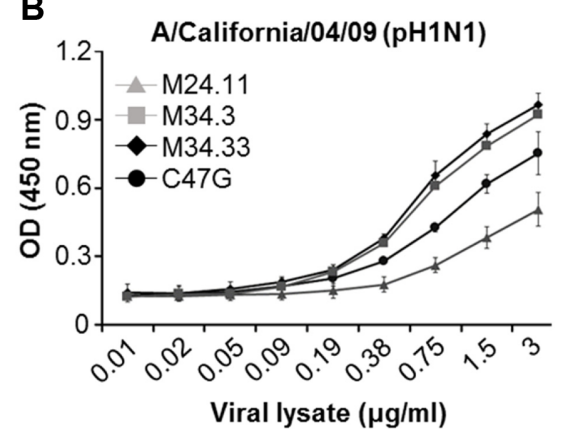

C

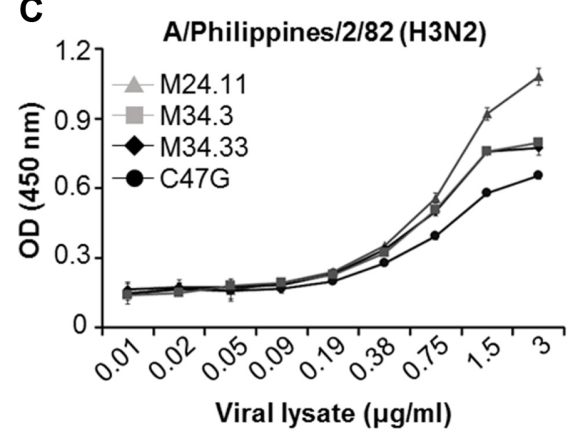

Fig. 4. Reactivity of anti-NP mAbs against influenza A viruses.

Viral lysates were prepared from A/Puerto Rico/8/34 (H1N1), A/California/04/09 (pH1N1), and A/Philippines/2/82 (H3N2). The lysates were coated at the indicated concentrations and the mAbs were applied to the plates at $100 \mathrm{ng} /$ well. The NP-specific mAb C47G was used for comparison. Corrected OD values are shown and are expressed as the mean \pm standard error.

\section{Evaluation of NP-Specific mAbs Using Influenza Virus-} Infected Tissues

To evaluate whether NP-specific mAbs can detect influenza virus in infected tissues, we challenged C57BL/6 mice intranasally with $10 \mathrm{LD}_{50}$ of H1N1. Four days postinfection, lung tissues were obtained. Sections were stained with the various mAbs and then incubated with Alexa Fluor 488-conjugated anti-mouse IgG. Fluorescent immunohistochemistry revealed that M24.11, M34.3, and M34.33 all effectively detected influenza virus in the infected lung sections. The commercial $\mathrm{mAb} \mathrm{C} 47 \mathrm{G}$ also recognized influenza virus in the lung sections (Fig. 5). Our results suggest that the generated anti-NP mAbs could be applicable for diagnosing the presence of influenza A viruses in infected tissues.

\section{Discussion}

In this study, we produced three mAbs that reacted with NP of influenza A viruses and tested whether they could be more sensitive in diagnosing influenza compared with the conventional anti-NP mAbs. Relative to the commercially available mAbs, our mAbs (M24.11, M34.3, and M34.33) all exhibited higher reactivities against recombinant NP antigen and influenza virus A/Puerto Rico/8/34 (H1N1). Two of them (M34.3 and M34.33) showed higher sensitivities to A/

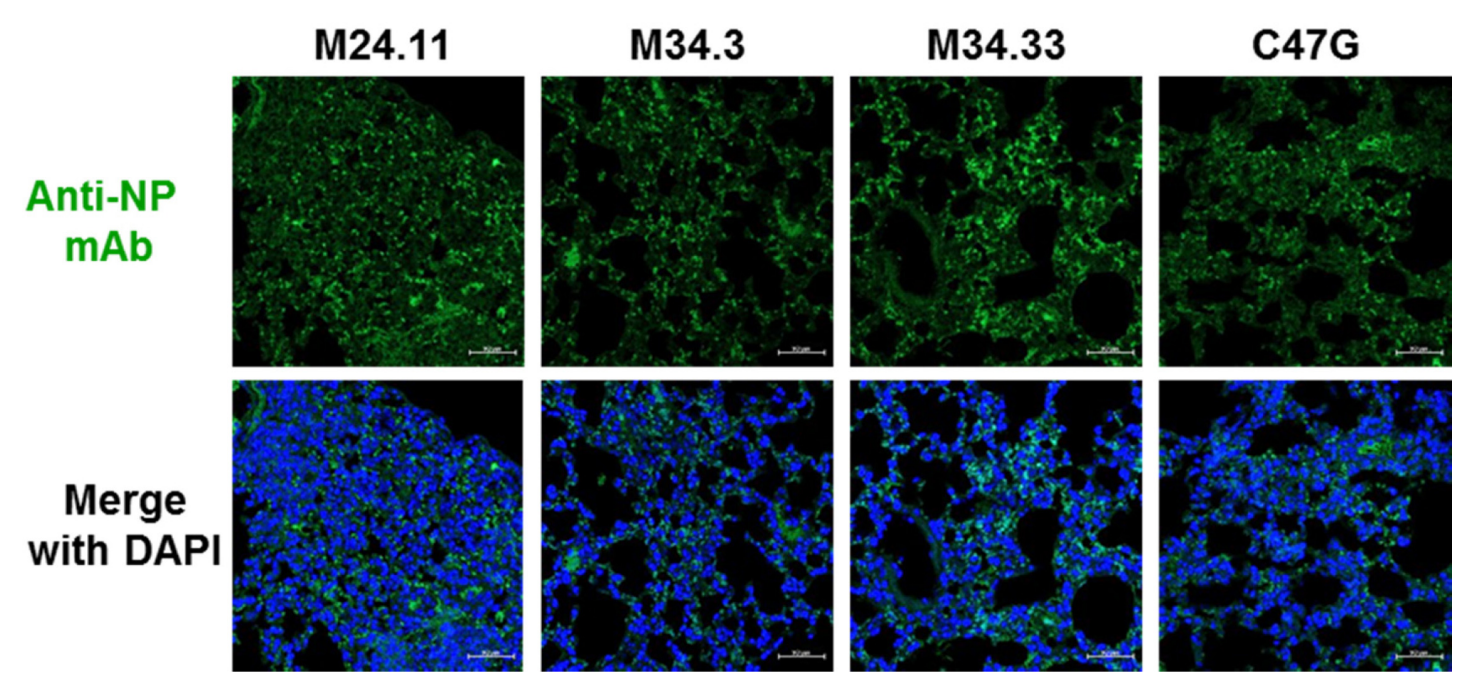

Fig. 5. Ability of the anti-NP mAbs to detect influenza A virus in infected tissues.

Mice were intranasally challenged with $10 \mathrm{LD}_{50}$ of $\mathrm{A} / \mathrm{PR} 8 / 34$ virus. Four days post-infection, lung tissues were harvested, fixed, paraffin embedded, and sectioned. The tissue sections were stained with the indicated mAbs and exposed to Alexa Fluor 488-conjugated anti-mouse IgG (green). Nuclei were counterstained with DAPI (blue). The NP-specific mAb C47G was used for comparison. 
California/04/09 (pH1N1) and A/Philippines/2/82 (H3N2) than the commercial $\mathrm{mAb}$. The third newly generated $\mathrm{mAb}, \mathrm{M} 24.11$, had lower reactivity to $\mathrm{pH} 1 \mathrm{~N} 1$ and higher reactivity to $\mathrm{H} 3 \mathrm{~N} 2$ compared with the commercial $\mathrm{mAb}$. These results indicate that M34.3 and M34.33 could be potentially useful for diagnosing influenza A viruses.

Anti-NP mAbs have been commonly used in proteinbased diagnostic strategies for influenza because NP has relatively well-conserved region among influenza A viruses [5]. Here, we found that mAbs M34.3 and M34.33 had drastically high reactivities to the influenza A viruses A/Puerto Rico/8/34 (H1N1), A/California/04/09 (pH1N1), and A/Philippines/2/82 (H3N2), whereas mAb M24.11 showed high reactivities to H1N1 and H3N2 but not pH1N1. As NP can show up to $95 \%$ sequence conservation among influenza A viruses at the protein level [20-22], our results suggest that M34.3 and M34.33 could react with more conserved epitopes than M24.11. Notably, when we performed western blot analysis under reducing conditions, rNP was detected by M24.11 but not by M34.3 and M34.33. In a previous study in which rNP antigens of various influenza A viruses were expressed using E. coli and insect cells, the authors found that NP forms oligomers via a flexible tail loop that is capable of inserting inside a neighboring molecule [23]. Antibodies used in ELISA can bind to native proteins by three-dimensional structure, but antibodies used in western blotting detect denatured proteins under reducing conditions. Thus, our present findings suggest that M34.3 and M34.33 recognize highly conserved conformational epitopes of NP of influenza A virus, and appear to be potentially effective for diagnosing influenza.

As the existing diagnostic kits detect influenza viruses in infected tissues of patients, we tested our newly generated NP-specific mAbs in this context. Fluorescent confocal microscopy of A/Puerto Rico/8/34 (H1N1) influenza virus-infected mouse lung tissue sections revealed that our newly developed $m A$ bs effectively detected influenza virus in the infected tissues. Thus, these mAbs may be appropriate for detecting influenza virus even within the current influenza diagnostic systems. In efforts to improve the sensitivity of influenza diagnosis, newly developed diagnostics have adopted various materials such as fluorescent dyes and nanoparticles [5]. However, the conjugation of any such material to an antibody can lead to its functional inactivation [24]. Further studies may be required to completely optimize the environments for use of an antibody for the diagnostic test.

In conclusion, we herein report the generation of new
mAbs that appear to be applicable for protein-based influenza diagnostics. M34.3 and M34.33 exhibited improved reactivity to influenza A viruses, compared with currently available $\mathrm{mAbs}$, and appeared to recognize highly conserved conformational epitopes of the influenza A NP. Seasonal and pandemic influenza pose ongoing risks to global health, and the accurate and rapid diagnosis of this disease is extremely important for its control. We believe that our newly developed mAbs could be used in diagnostic kits that seek to confirm influenza virus infections.

\section{Acknowledgments}

This work was supported by grants from the R\&D Convergence Program of National Research Council of Science \& Technology (No. CAP-16-02-KIST) and the National Research Foundation of Korea (No. NRF-2016M3A9B6918584).

\section{Conflict of Interest}

The authors have no financial conflicts of interest to declare.

\section{References}

1. Webster RG, Bean WJ, Gorman OT, Chambers TM, Kawaoka Y. 1992. Evolution and ecology of influenza A viruses. Microbiol. Rev. 56: 152-179.

2. Cox NJ, Subbarao K. 1999. Influenza. Lancet 354: 1277-1282.

3. Neumann G, Noda T, Kawaoka Y. 2009. Emergence and pandemic potential of swine-origin H1N1 influenza virus. Nature 459: 931-939.

4. Molinari NA, Ortega-Sanchez IR, Messonnier ML, Thompson WW, Wortley PM, Weintraub E, et al. 2007. The annual impact of seasonal influenza in the US: measuring disease burden and costs. Vaccine 25: 5086-5096.

5. Vemula SV, Zhao J, Liu J, Wang X, Biswas S, Hewlett I. 2016. Current approaches for diagnosis of influenza virus infections in humans. Viruses 8: 96.

6. Kumar S, Henrickson KJ. 2012. Update on influenza diagnostics: lessons from the novel H1N1 influenza A pandemic. Clin. Microbiol. Rev. 25: 344-361.

7. Uyeki TM. 2003. Influenza diagnosis and treatment in children: a review of studies on clinically useful tests and antiviral treatment for influenza. Pediatr. Infect. Dis. J. 22: 164-177.

8. Falsey AR, Murata Y, Walsh EE. 2007. Impact of rapid diagnosis on management of adults hospitalized with influenza. Arch. Intern. Med. 167: 354-360.

9. Mizuike R, Sasaki T, Baba K, Iwamoto H, Shibai Y, Kosaka M, et al. 2011. Development of two types of rapid diagnostic 
test kits to detect the hemagglutinin or nucleoprotein of the swine-origin pandemic influenza A virus H1N1. Clin. Vaccine Immunol. 18: 494-499.

10. Yang M, Berhane Y, Salo T, Li M, Hole K, Clavijo A. 2008. Development and application of monoclonal antibodies against avian influenza virus nucleoprotein. J. Virol. Methods 147: 265-274.

11. Drexler JF, Helmer A, Kirberg H, Reber U, Panning M, Muller M, et al. 2009. Poor clinical sensitivity of rapid antigen test for influenza A pandemic (H1N1) 2009 virus. Emerg. Infect. Dis. 15: 1662-1664.

12. Uyeki TM, Prasad R, Vukotich C, Stebbins S, Rinaldo CR, Ferng YH, et al. 2009. Low sensitivity of rapid diagnostic test for influenza. Clin. Infect. Dis. 48: e89-e92.

13. Linke S, Neubauer K, Dorner MB, Dorner BG, Pauli G, Schweiger B. 2011. Generation and characterisation of monoclonal antibodies against influenza virus A, subtype H5N1. J. Virol. Methods 175: 85-94.

14. Wang W, Huang B, Jiang T, Wang X, Qi X, Gao Y, et al. 2012. Robust immunity and heterologous protection against influenza in mice elicited by a novel recombinant NP-M2e fusion protein expressed in E. coli. PLoS One 7: e52488.

15. Yang J, Shim SM, Nguyen TQ, Kim EH, Kim K, Lim YT, et al. 2017. Poly-gamma-glutamic acid/chitosan nanogel greatly enhances the efficacy and heterosubtypic cross-reactivity of H1N1 pandemic influenza vaccine. Sci. Rep. 7: 44839.

16. Heo CK, Woo MK, Yu DY, Lee JY, Yoo JS, Yoo HS, et al. 2010. Identification of autoantibody against fatty acid synthase in hepatocellular carcinoma mouse model and its application to diagnosis of HCC. Int. J. Oncol. 36: 1453-1459.

17. Shaw ML, Stone KL, Colangelo CM, Gulcicek EE, Palese P. 2008. Cellular proteins in influenza virus particles. PLoS Pathog. 4: e1000085.

18. Heyward JT, Klimas RA, Stapp MD, Obijeski JF. 1977. The rapid concentration and purification of influenza virus from allantoic fluid. Arch. Virol. 55: 107-119.

19. Polson A, Keen A, Sinclair-Smith C, Furminger IG. 1972. Polyethylene glycol purification of influenza virus with respect to aggregation and antigenicity. J. Hyg. (Lond.) 70: 255-265.

20. Shu LL, Bean WJ, Webster RG. 1993. Analysis of the evolution and variation of the human influenza A virus nucleoprotein gene from 1933 to 1990. J. Virol. 67: 2723-2729.

21. Reid AH, Fanning TG, Janczewski TA, Lourens RM, Taubenberger JK. 2004. Novel origin of the 1918 pandemic influenza virus nucleoprotein gene. J. Virol. 78: 12462-12470.

22. Babar MM, Zaidi NU. 2015. Protein sequence conservation and stable molecular evolution reveals influenza virus nucleoprotein as a universal druggable target. Infect. Genet. Evol. 34: 200-210.

23. Ye Q, Krug RM, Tao YJ. 2006. The mechanism by which influenza A virus nucleoprotein forms oligomers and binds RNA. Nature 444: 1078-1082.

24. Werthen M, Nygren H. 1988. Effect of antibody affinity on the isotherm of antibody binding to surface-immobilized antigen. J. Immunol. Methods 115: 71-78. 